\title{
Techno-economic analysis for the deployment of PPDR services over 4G/4G+ Networks
}

\author{
Theofilos Chrysikos. Panagiotis Galiotos, Stavros \\ Kotsopoulos \\ Dept of Electrical and Computer Engineering \\ University of Patras \\ Patras, Greece \\ Email: txrysiko@ece.upatras.gr,pgaliot@upatras.gr, \\ kotsop@ece.upatras.gr
}

\author{
Tasos Dagiuklas \\ School of Science \& Technology \\ Hellenic Open University \\ Patras, Greece \\ Email: dagiuklas@eap.gr \\ http://cones.eap.gr
}

\begin{abstract}
This paper presents a techno-economic study and sensitivity analysis for the deployment of PPDR services over next-generation networks. More specifically, the deployment of a core LTE network is investigated for providing PPDR services over $700 \mathrm{MHz}$ for an urban scenario with severe shadow fading conditions. The service limits for user and bandwidth congestion for this network are examined and a backhaul solution at the microwave band is proposed via small cells. Techno-economic parameters are taken into consideration and both basic sensitivity analysis and multivariate simulations are presented to establish the robustness of network performance for certain channel and user conditions.
\end{abstract}

Keywords-techno-economic analysis; PPDR; 4G; backhaul; sensitivity analysis

\section{INTRODUCTION}

Public Protection and Disaster Relief (PPDR) services have attracted both academia and industry in an effort to enhance the existing Private Mobile Radio (PMR) solutions [1]-[3] all over Europe and globally in order to incorporate the benefits of emerging wireless broadband technologies such as Long-Term Evolution (LTE) and LTE-Advanced (LTE-A) [4]-[8]. Whereas technologies such as Terrestrial Trunked Radio (TETRA/TETRAPOL) [9]-[13] operate in the UHF frequency and allow for the exploitation of better propagation conditions in those bands, namely lower distance-dependent path loss [14], they still have to struggle with limited spectrum and interference from other systems such as television (TV) broadcasting [15]. On the other hand, microwave frequencies offer higher bandwidth but also more severe attenuation due to shadow fading and other phenomena [16].

In the context of the SALUS project (Security and interoperability in next generation PPDR communication infrastructures) [17], the implementation of a "macro" LTE network has been investigated in the $700 \mathrm{MHz}$ channel (band 28 ) in order to take advantage of the propagation conditions compared to microwave sub- $6 \mathrm{GHz}$ frequencies, and, at the same time, allow for sufficient bandwidth and throughput for the network users. By considering a variable but upper-bound number of authenticated users such as rescue workers and other personnel of specific nature, an optimal dimensioning of various topologies of interest, not limited to but certainly focused in urban and densely-populated areas can allow for a technically robust deployment and a cost-efficient solution for interested providers, be it private investors or public-state authorities.

In such a process, the techno-economic analysis is of critical importance to the assessment and evaluation of all involved costs, be it the primary investment or the operational expenditure in the depth of a certain time period, i.e. a few years. Trends in yearly costs need to be considered and investigated accordingly. A number of published works has studied different scenarios of both Ultra High Frequency (UHF) and microwave band deployment of broadband networks as well as backhaul solutions and has provided an insight on the investment and operational cost for different throughput and service requirements [18]-[24].

Our work features the presentation of a respective technoeconomic tool that has been developed for this purpose, as well as basic and multivariate sensitivity analysis for the technical parameters, including various propagation scenarios, different case studies of the urban surface where the LTE deployment must be applied and a variable number of rescue workers and authenticated users that need to access the network with certain throughput requirements. We focus primarily on the channel conditions and thoroughly investigate the critical impact of different shadow (large-scale) fading scenarios on the LTE deployment, service delivery and subsequent economic figures. Our findings can be applied both to "macro" LTE networks deployed for security scenarios as well as for backhaul provision of security and safety services by obtaining spectrum and resources from other operating core networks.

The paper is structured as follows: Section II presents the techno-economic tool and the fundamental concept of the LTE PPDR network, as well as the methodology employed in the tool and the various steps the user can take in order to retrieve information about hardware demands and the logistics of the deployment. Section III presents a basic and a multivariate analysis of the technical parameters involved in the network deployment. Section IV provides a fundamental economic analysis with specific yearly trends in operational expenditure. Section $\mathrm{V}$ discusses the results and conclusions and presents the issues that need to be addressed in immediate future work. 


\section{TECHNO-ECONOMIC TOOL}

The Techno-Economic Tool (TE Tool) for PPDR services has been developed in the context of the SALUS European Research Project. It introduces the idea of merging several tools that can assist with the planning and dimensioning of a PPDR Network, based on a multitude of available technologies and the appropriate configuration. Subsequently, the TE Tool directs the user on how to perform a basic technoeconomic analysis in order to examine the value and the viability of the deployment of specific technologies, in a shorter or longer term of duration.

Furthermore, the TE Tool can compare the technoeconomic behavior of different deployment scenarios, in a time period of up to 7 years. Each scenario implements an existing technology for designing a PPDR network. The user can input the required data in a multitude of ways and the results are also presented either in a graphic format or as a downloadable excel file. More information about the tool can be found in [25]. Fig.1 depicts the tool's interface.

The LTE Dimensioning tool assists the user to decide on the actual planning of a PPDR network, the coverage area and the number of base stations, based on the existing LTE technology. The calculations are provided in the context of SALUS project. Hence, the user is initially asked to decide on the required coverage area by choosing between the three, predefined scenarios of the SALUS architecture, the Urban, the Suburban and the Crowded Event. The tool differentiates appropriately the signal fading, based on the characteristics of the user's choice.

Our aim is to investigate the impact of propagation intrinsic characteristics on the deployment of the PPDR services in a certain frequency in order to provide an estimation, robust to the best possible degree, of the hardware equipment and subsequent cost, and its service limitations in terms of the number of users that can be simultaneously accessing this LTE network for a given bit rate. Our scenario includes the possible extension of the LTE PPDR network via backhaul support at microwave or mm-wave frequencies, depending on the channel conditions. NLOS scenarios have been investigated in the following schemes for microwave backhaul already supported by providers, as a backhaul extension based on bit rate demand, as shown in Fig.2.

\section{SALUS}

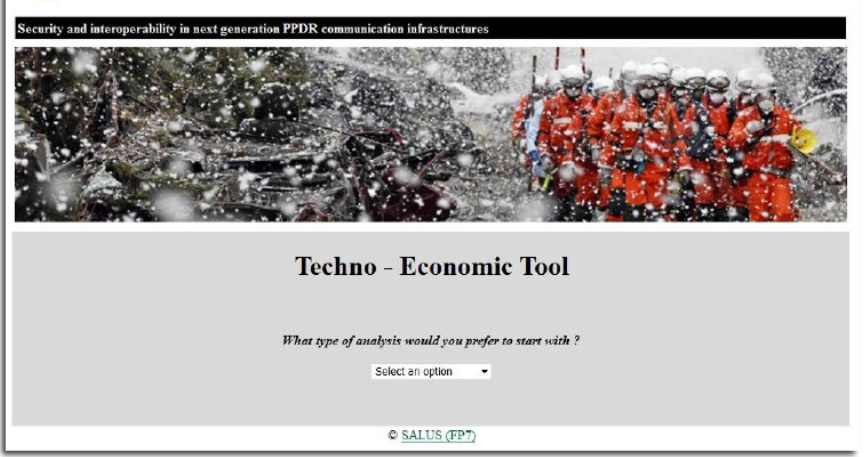

Fig. 1. Techno-economic tool interface

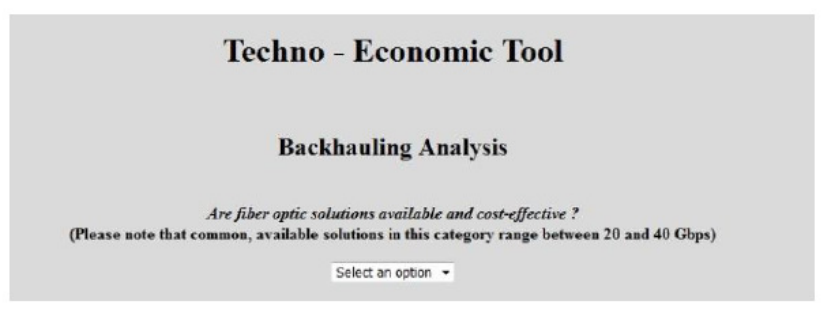

Fig. 2. Backhaul analysis

Initially, the Fiber Optics is examined as the optimal solution. However, if due to the cost this cannot be an option, the TE Tool proceeds to suggesting the Sub- $6 \mathrm{GHz}$ licensed solution product for implementing the backhaul network. If the capacity is adequate, then a NLOS Sub-6 GHz licensed product can be examined. Otherwise, LOS products can be considered. If microwave/millimeter spectrum is available, then this should be chosen as the LOS solution, otherwise the option of achieving LOS through hop relay should be considered. If this option is also undesired, then a NLOS Sub$6 \mathrm{GHz}$ unlicensed solution should be considered, otherwise the fiber optic idea can be re-visited, despite the cost. The user is guided through the algorithm and certain hints regarding available prices and capacity are presented.

Having completed the dimensioning part, the PPDR user can proceed to the techno-economic analysis, as shown in Fig. 3 and 4.

\section{SENSITIVITY ANALYSIS}

\section{A. Basic Parameters for Deployment}

In order to evaluate and confirm the robustness of the techno-economic tool, it is imperative to conduct both basic and multivariate sensitivity analysis of the necessary LTE deployment for the selected frequency band of $700 \mathrm{MHz}$. This sensitivity analysis will involve technical parameters that investigate the service limitations from a standpoint of both propagation effects and large-scale (shadow) fading due to obstacles and buildings and limitations from a bit rate demand for all rescue workers authenticated as PPDR network users.

For the urban scenario in question, a local-range LTE BS with a total (maximum) output power of $24 \mathrm{dBm}$, [4] is considered. The city scenario employs a variety of rescue workers whose number and location depends on the emergency scenario and the nature of a possible incident. In addition, the area needed to be covered by the LTE BSs depends significantly on whether this is a daily emergency case or an extreme emergency scenario.

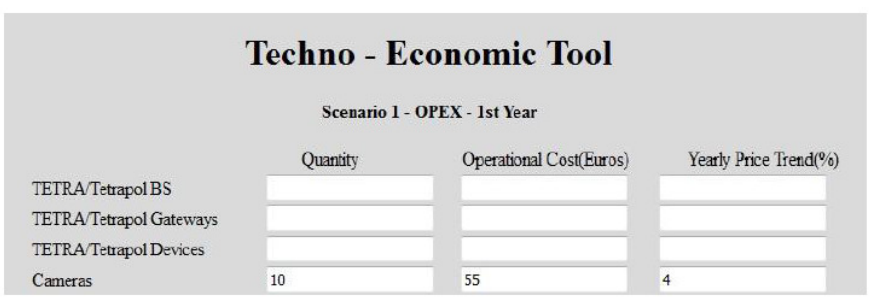

Fig. 3. Opex analysis for various technologies 


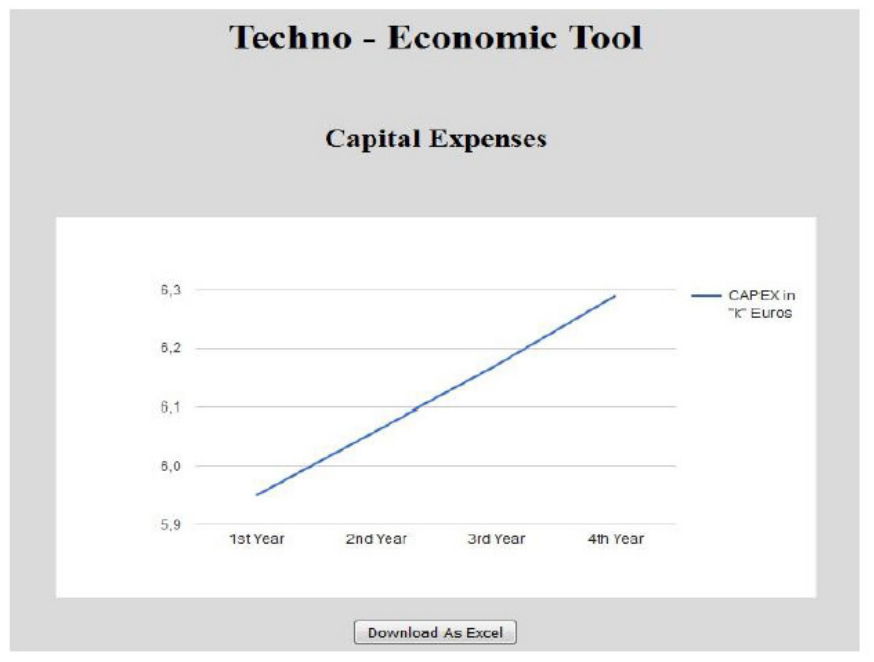

Fig. 4. Techno-economic analysis of capital expenses over a 4-year period

Considering the propagation characteristics and subsequent physical range limitations, the coverage distance $d(m)$ for each LTE BS operating at $700 \mathrm{MHz}$ is provided by:

$d(m)=10^{\left[\frac{P_{t}(d B m)+G_{t}(d B)-P_{R}(d B m)+27.55-20 \log _{10} f(M H z)-(1.645 \times \sigma(d B))}{20}\right]}$

Where: $P_{t}(d B m)$ is the transmitted power, $G_{t}(d B)$ the transmitting antenna gain, $P_{r}(\mathrm{dBm})$ the received signal threshold, $f(M H z)$ the carrier frequency band $(700 \mathrm{MHz}$ for the specific LTE PPDR network) and $\sigma(d B)$ shadow deviation (depth) for the topology in question. The basic sensitivity analysis focuses on the shadow (large-scale) fading while assuming pre-fixed values for the other variables.

We consider a received power level of $-80 \mathrm{dBm}$, allowing for at least $8 \mathrm{~dB}$ of SNR at cell-edge boundary conditions. The interference levels are assigned at $-88 \mathrm{dBm}$ since this is an interference-bound channel due to TV broadcasting, a value provided by extensive outdoor measurements conducted with the NARDA SRM-3006 measuring equipment in the urban centers of Athens, Greece and Patras, Greece [26].

Therefore, the critical distance/radius of each LTE BS is found to be:

$$
d(m)=10^{\left[\frac{74.65 d B-(1.645 \times \sigma(d B))}{20}\right]}
$$

Clearly, this is a shadow fading-based condition for the cell boundaries. Since this is an urban scenario, the city is expected to be dense with buildings and other obstacles of significant size compared to the wavelength. Thus, typical shadow deviation values in a severely obstructed environment according to research are assumed to range between 6 and 14 $\mathrm{dB}$ [27]. Taking a worst case approach of $14 \mathrm{~dB}$, the critical distance is found to be equal to approximately 380 meters (381 meters).

According to [28], an SNR of $8 \mathrm{~dB}$ provides a bit rate of $40 \mathrm{Mbps}$, if a bandwidth of $20 \mathrm{MHz}$ is considered for our LTE system. This has been provided by simulations conducted when an urban macro-cell scheme is considered. By macrocell, the authors in [28] define a scenario of urban propagation where the radio-channel eigenvalues are calculated independently from the cell deployment (being linked to the SNR and BS clustering and sectoring) [29]. This applies to our case as well, where the deployment of the LTE BS is directly derived from a critical coverage distance based on an areamean shadow depth that does not account for the specific characteristics and radio environment of each cluster but considers a given SNR based on a local received power threshold (and an average level of unwanted, interfering signal).

Therefore, the radio channel eigenvalues can be calculated independently from the cell deployment strategy and given a certain SNR (8 dB in our case), an expected bit rate can be found, as already mentioned, equal to $40 \mathrm{Mbps}$.

If $B$ is the total downlink rate and $b$ the expected average bit rate of each user, then the number of users at the boundary of the area of coverage for each BS which can be serviced with the pre-defined user bitrate $\mathrm{b}$ equals $\mathrm{B} / \mathrm{b}$. If $2 \mathrm{Mbps}$ are required per user (marginal broadband connectivity), then a total of 40 users can be serviced per average by one LTE BS of the PPDR network. For 200 rescue workers, corresponding to an average number of deployed police officers, traffic patrol officers, first aid workers and stand-by firefighters in a daily emergency scenario, a total of 5 LTE BS are needed. This corresponds to a total surface of 2.8 square kilometers.

If an area of $\mathrm{x}$ meters (length) and y meters (width) is considered as the input data instead of a specific number of users, then the number of LTE BSs is calculated by:

$$
z=\frac{x(m) \times y(m)}{4 \times d^{2}}
$$

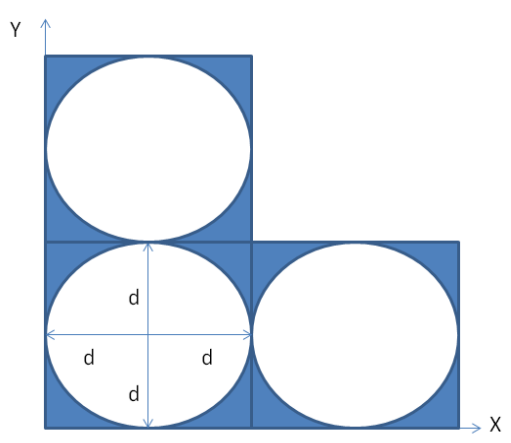

Fig. 5. Square approximation of LTE cell for the urban scenario 
The typical coverage scenario of an omni-directional BS considers a full circle of $2 \pi$ and radius equivalent to the maximum T-R separation (d_coverage) so that the local mean value of the received power will not drop below the predefined threshold ( $-80 \mathrm{dBm}$ in our case). In order to simply our calculations, a square coverage scenario will replace the circular one (still considering omni-directional directivity). Fig. 5 provides a comparison of circular versus square approximation in terms of coverage. This approach leaves no "white areas" between different BSs of the LTE infrastructure.

TABLE I. COVERAGE DISTANCE FOR VARIOUS SHADOWING SCENARIOS

\begin{tabular}{|c|c|c|c|}
\hline Shadow & \multicolumn{3}{|c|}{ Maximum coverage distance(m) } \\
\hline$\sigma(\mathrm{dB})$ & $\operatorname{Pr}=-75 \mathrm{dBm}$ & $\operatorname{Pr}=-80 \mathrm{dBm}$ & $\operatorname{Pr}=-85 \mathrm{dBm}$ \\
\hline 2 & 2080 & 3698 & 6577 \\
\hline 4 & 1424 & 2532 & 4503 \\
\hline 6 & 975 & 1734 & 3083 \\
\hline 8 & 668 & 1187 & 2111 \\
\hline 10 & 457 & 813 & 1445 \\
\hline 12 & 313 & 557 & 990 \\
\hline 14 & 214 & 381 & 678 \\
\hline
\end{tabular}

In order to provide a thorough basic sensitivity analysis, a shadow depth $\sigma(\mathrm{dB})$ of ranging values from 0 (LOS) to $14 \mathrm{~dB}$ (severe obstacle shadowing) has been assumed. Three different received power levels have been considered: our predefined threshold of $-80 \mathrm{dBm}$, the $-85 \mathrm{dBm}$ threshold recommended by the LTE specifications [4] and a "stricter" received power requirement of $-75 \mathrm{dBm}$. Results are depicted in Fig. 6 and some indicative values are shown in Table I.

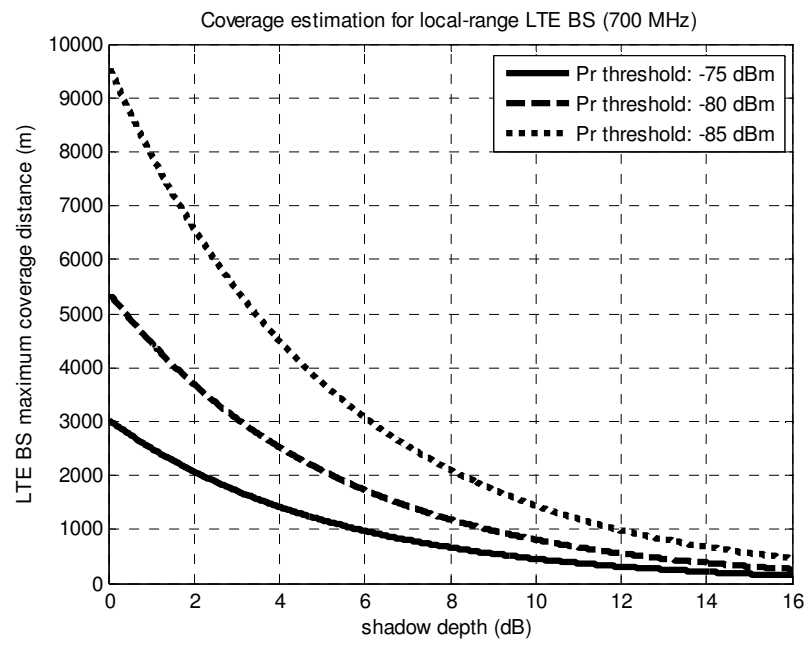

Fig. 6. Urban scenario of coverage estimation for LTE at $700 \mathrm{MHz}$ (band 28)
Whereas results deviate more in the LOS-region of small shadow deviation values, differences between the three scenarios remain evident even in the more shadowed case studies as well. The original LTE specifications are more idealistic in terms of interference and excess path loss due to shadow fading from obstacles and buildings. The $-75 \mathrm{dBm}$ threshold is largely impractical due to its excessive severity and is presented for the sake of completeness, in the case where a sudden increase in interference levels provides the necessity for a stricter threshold.

For an extreme emergency scenario where the number of rescue workers increases, a cost-effective approach would be to lease spectrum from a Mobile Network Operator (MNO) in order to provide backhaul support to the PPDR LTE network. Since extreme emergency scenarios correspond to conditions occurring within a very short time period, it is not viable to include these abrupt increases in bit rate within the dimensioning of the LTE PPDR network, but to provide an on-the-fly, reliable access to resources from MNOs in order to meet with these bursts in demand.

\section{B. Multivariate sensitivity analysis}

The multivariate sensitivity analysis investigates all possible outcomes of the involved variables, namely the shadow depth, the surface of the urban area designed for coverage by the LTE PPDR network, the average SNR and the number of users authorized to access the PPDR services.

The multivariate analysis thus investigates the boundaries and statistical behavior of the number of LTE BSs that need to be deployed in order to provide coverage in an urban area with certain (or varying) shadowing conditions, and the downlink bit rate per user (Mbps).

The shadow depth varies as in the basic analysis from o to $14 \mathrm{~dB}$, covering all shadow fading scenarios from near-perfect LOS to heavy obstruction (NLOS). The limit of the urban surface designated for coverage has been set to 50 square kilometers. Results are shown in Fig. 7, where in the worst scenario of $14 \mathrm{~dB}$ and 50 square kilometers, less than 90 LTE BSs are required.

Fig. 8 demonstrates the bit rate per user (Mbps) with average user SNR and number of users as input parameters. An average receiver SNR ranging from $8 \mathrm{~dB}$ (cell-edge requirement as stated in the previous section) up to $20 \mathrm{~dB}$ has been considered. According to [28]-[29], with an SNR of 20 $\mathrm{dB}$, the upper limit of $96 \mathrm{Mbps}$ (simulation value as opposed to the theoretical boundary of $100 \mathrm{Mbps}$ ) is already achieved for the LTE protocol, therefore higher SNR values are of little interest since they do not influence the bit rate value any further. The number of rescue workers is also investigated, as already mentioned, with a maximum of 250 (per cell). Though this is a value larger than in most realistic scenarios, it is still possible to meet such crowded cells in an extreme emergency scenario. It is, therefore, important to investigate these values of user density. 


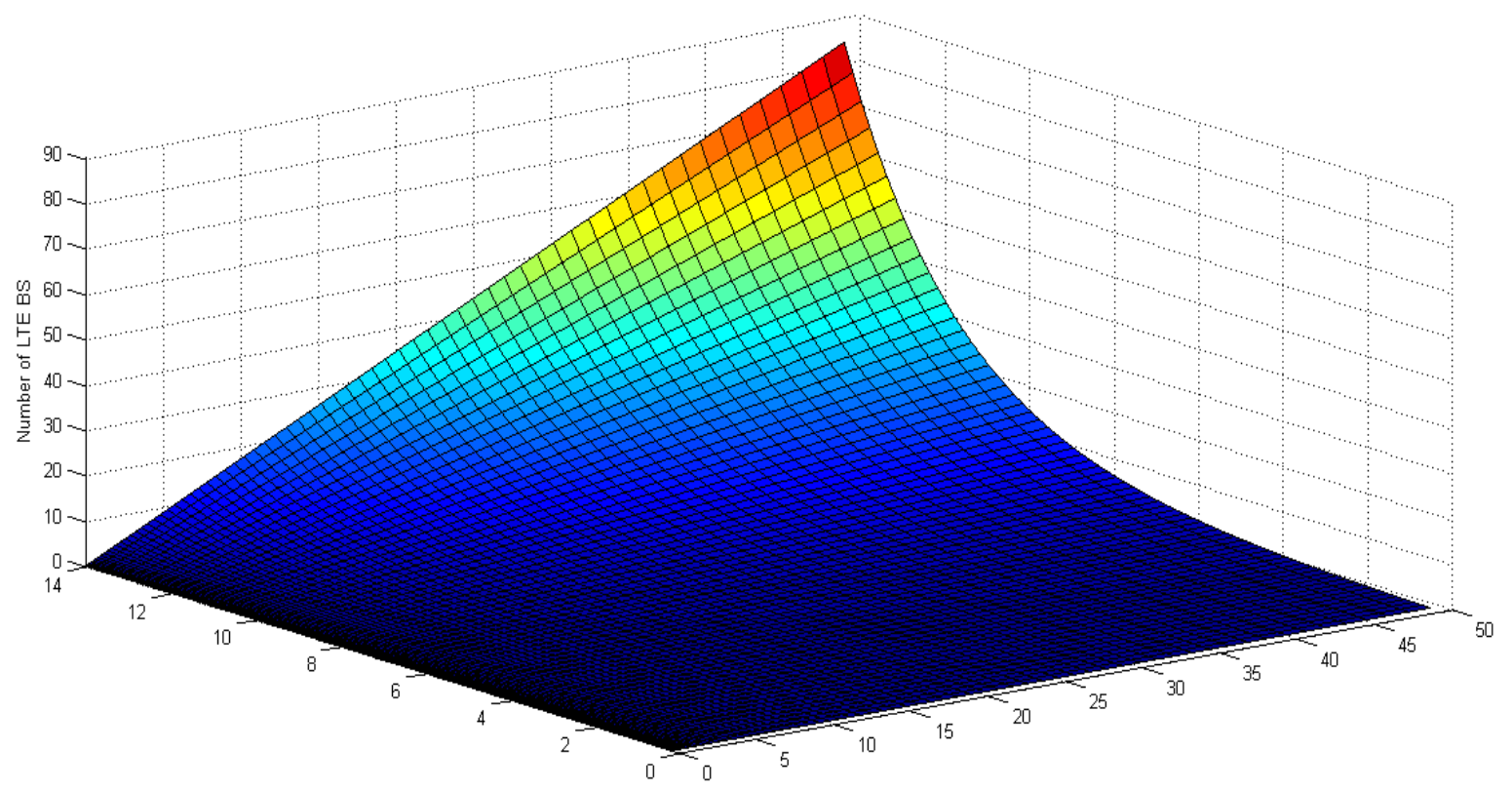

shadow depth (dB)

Urban surface for coverage (sq. km)

Fig. 7. Number of LTE BS for various shadow fading and urban surface scenarios

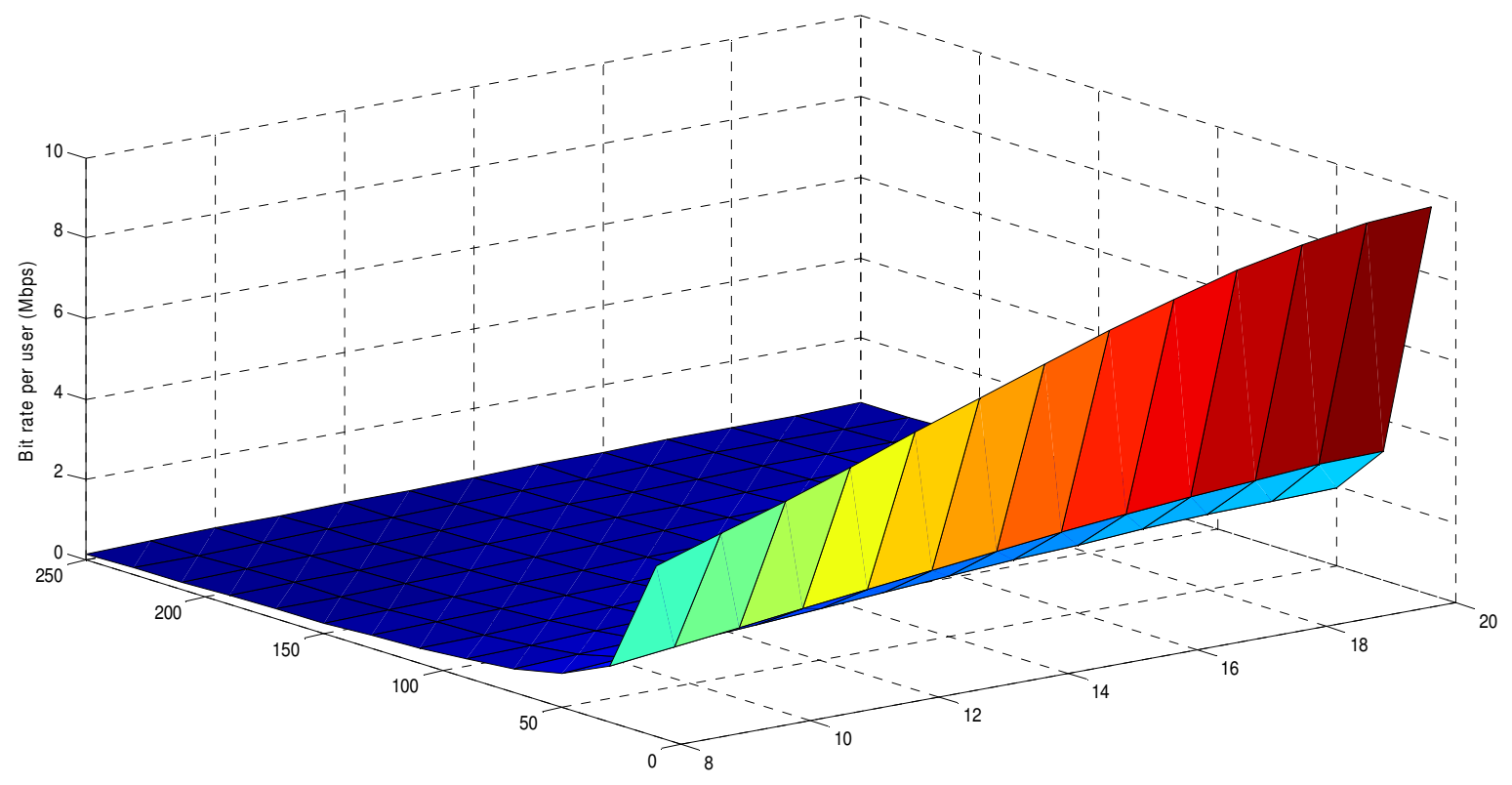

rescue workers per cell

Average SNR $(\mathrm{dB})$

Fig. 8. Bit rate per user for various schemes of receiver SNR and number of rescue workers per cell 


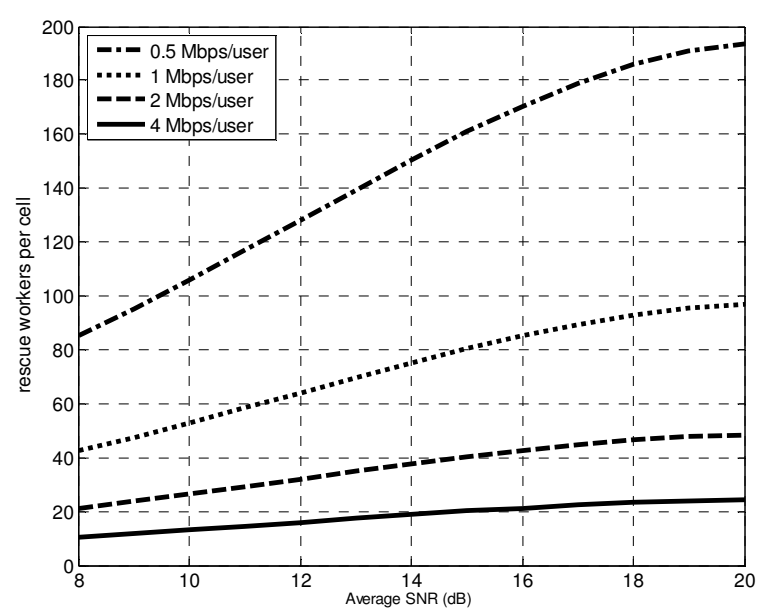

Fig. 9. Maximum number of users per cell for various SNR schemes and predefined bit rate (per user) requirements

Fig. 9 depicts the maximum number of users per cell when certain requirements concerning the expected bit rate per user have been set, ranging from 0.5 to $4 \mathrm{Mbps}$ per user, for various SNR schemes. The upper bounds are examined and the limitations from both propagation and throughput are derived.

It is obvious that the bit rate demand per user drastically affects the average (and maximum) number of rescue workers per cell, with mean values of 146, 73, 36 and 18 for $0.5,1,2$ and $4 \mathrm{Mbps}$ per user respectively. This provides in all cases, an average total downlink bit rate of roughly $72-73 \mathrm{Mbps}$. The average SNR is considered to be $14 \mathrm{~dB}$, which corresponds to an average user location at the half of the cell radius, aside from the fact that for SNR values higher than $20 \mathrm{~dB}$, the total downlink bit rate does not exceed theoretical and realistic bounds (100 Mbps and 96 Mbps respectively).

\section{CAPEX AND OPEX ANALYSIS}

In terms of both capital and operational expenditure (Capex and Opex respectively), the economic analysis takes into consideration both the investment amount for the LTE BS deployment and installation, as well as operational costs running yearly with a $17.5 \%$ ratio. By applying these costrelated data [22] to the dimensioning that has been investigated in the previous sections, we can calculate approximately the total cost over, i.e. a six-year period since the first installation of the LTE BSs required for a given urban surface.

We study various channel conditions that correspond in different propagation scenarios ranging from near-LOS $(2 \mathrm{~dB})$ to worst-case severe shadow fading due to buildings and obstacles (a common occurrence in metropolitan centers as well as in topologies where a natural or man-made disaster has left a lot of ruins, obstacles and debris). Results are shown in Fig. 10. The urban surface can vary from a few square kilometers up to 50. Mean costs range from 67650 Euros for 2 $\mathrm{dB}$ shadow depth (1 LTE BS needed), up to approximately 3 million Euros for worst-case $14 \mathrm{~dB}$ shadow fading.

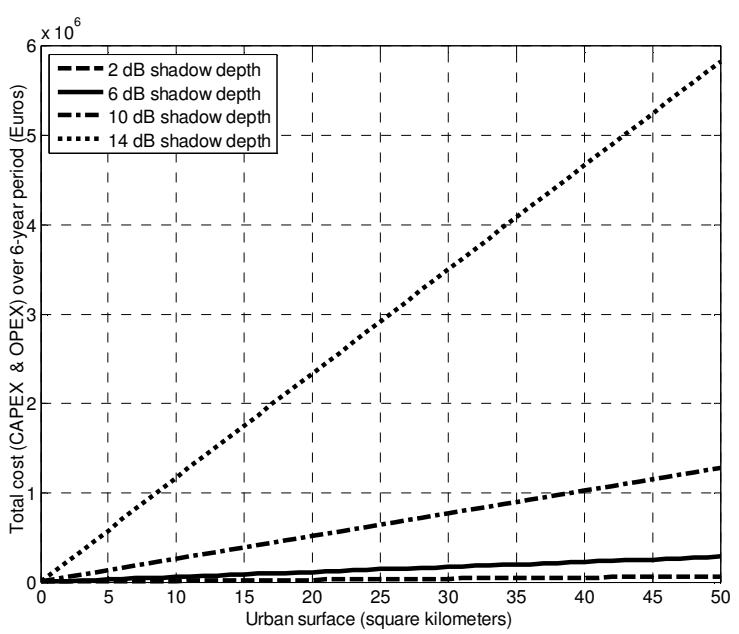

Fig. 10. Total cost (Capex \& Opex) for various channel conditions and variable urban surface over a six-year period

\section{CONCLUSION AND FUTURE WORK}

In this work, the deployment of an LTE network for PPDR services was investigated, for band 28 and an urban environment. A techno-economic tool was designed to provide on-the-fly assessment of the equipment and logistics involved with the installation and operation of a PPDR network. The robustness of the tool was evaluated by conducting both basic and multivariate sensitivity analysis for the $700 \mathrm{MHz}$ channel and an urban topology scenario for various shadow fading conditions and for a variable number of rescue workers with different bit rate requirements and limitations.

Realistic values for interference lead to specific SNR thresholds at the cell boundaries of each LTE BS, influencing the number of required equipment and the restrictions upon the total downlink bit rate. Multivariate analysis allows for the statistical analysis of the deployment and service throughput when more than one condition is examined for a pre-defined range of values.

Since all of the involved parameters are of technical nature, either representing propagation and fading impairments or spectral efficiency and throughput bounds, the need for a respective analysis and evaluation of economic parameters is needed. This is of critical importance not only for the LTE PPDR network but also for a possible extension towards $4 \mathrm{G}+$, since there might be, in our scenario, a possible need for extension of the provided PPDR services via backhaul support at microwave or mm-wave frequencies, depending on the channel conditions and the additional bit rate requirements.

As part of immediate future work, Monte-Carlo simulations will be conducted in order to derive the costanalysis for the long-term impact of the technical parameters on the viability of a $4 \mathrm{G} / 4 \mathrm{G}+$ combined system, by considering various schemes of yearly trend and its impact on the variability of operational expenditure. Moreover, other technologies, such as $802.11 \mathrm{n}$, will be studied in terms of both core network deployment and potential backhaul solution. 


\section{ACKNOWLEDGMENT}

This work was supported by the SALUS project (grant number: 313296), funded by the EC FP7 ICT-security collaborative research.

\section{REFERENCES}

[1] "User requirements and spectrum needs for future European broadband PPDR systems (Wide Area Networks)," Report 199, ECC, 20-24 May 2013.

[2] J. Marcus, J. Burns, V. Jervis, R. Wahlen, K. Carter, and I. Philbeck, "PPDR Spectrum Harmonisation in Germany, Europe and Globally," Report, WIK-Consult, 2010.

[3] A. P. Avramova, S. Ruepp, and L. Dittmann, "Towards future broadband public safety systems: Current issues and future directions", 2015 International Conference on Information and Communication Technology Convergence (ICTC), pp. 74 - 79.

[4] 3GPP TS36.104 version 11.8.2. R11.

[5] F. Liberal, J. Fajardo, N. Goia and I. Mesogiti, "Global standards, the key enablers for deploying next generation emergency communications networks", Proceedings of the 2014 ITU Kaleidoscope Academic Conference: Living in a converged world - Impossible without standards?, pp. 143-150.

[6] M. Manic, "Next generation emergency communication systems via software defined networks", 2014 Third GENI Research and Educational Experiment Workshop (GREE), pp. 1-8.

[7] K. Gomez, L. Goratti, T. Rasheed and L. Reynaud, "Enabling disasterresilient 4G mobile communication networks", Communications Magazine, IEEE, vol. 52, no. 12, pp. 66-73, 2014.

[8] A. Paulson and T. Schwengler, "A review of public safety communications, from LMR to voice over LTE (VoLTE)", 2013 IEEE 24th International Symposium on Personal, Indoor and Mobile Radio Communications (PIMRC), pp. 3513-3517.

[9] A. Salkintzis, "Evolving Public Safety Communication Systems by Integrating WLAN and TETRA Networks", IEEE Communications Magazine, vol. 44, no. 1, pp. 38-46, 2006.

[10] R. R. M. Group, "Phil Godfrey's views on TETRAs Future", 2015.

[11] "EN 300 392-2 v2.3.10", Terrestrial Trunked Radio (TETRA); Voice plus Data (V+D); Part 2: Air Interface (AI), 2003.

[12] "EN 300 392-7 v2.1.1", Terrestrial Trunked Radio (TETRA); Voice plus Data (V+D); Part 7: Security, 2001.

[13] "TR 102 021-4", Terrestrial Trunked Radio (TETRA); User Requirement Specification TETRA Release 2; Part 4: Air Interface Enhancements, 2004.

[14] W. C. Jakes (Ed.), Microwave mobile communications. New York, NY: Wiley Interscience, 1974.

[15] J. Seybold, Introduction to RF Propagation. Hoboken, NJ: Wiley Interscience, 2005.

[16] T. Rappaport, Wireless Communications: Principles \& Practice. Upper Saddle River, NJ: Prentice Hall, 1999.

[17] https://www.sec-salus.eu/

[18] T. Frisanco, P. Tafertshofer, P. Lurin and R. Ang, "Infrastructure Sharing for Mobile Network Operators; From a Deployment and Operations View", Munich, 2008.

[19] A. Khan, W. Kellere, K. Kozu and M. Yabusaki, "Network sharing in the next mobile network: TCO reduction, management flexibility, and operational independence", IEEE Communications Magazine, vol. 49, no. 10, pp. 134-142, 2011.

[20] J. Markendahl, Mobile Network Operators and Cooperation - A Teleeconomic study of infrastructure sharing and mobile payments services, Stockholm: Ph.D dissertation, CoS, KTH, 2011.

[21] S. Landström, A. Furuskär, K. Johansson, L. Falconetti and F. Kronestedt, "Heterogeneous networks - increasing cellular capacity", Ericsson review, No 2, 2011.
[22] Z. Frias and J. Pérez, "Techno-economic analysis of femtocell deployment in long-term evolution networks," EURASIP Journal on Wireless Communications and Networking, p. 288, 2012.

[23] J. Markendahl and M. Nilson, "Business models for deployment and operation of femtocell networks; - Are new cooperation strategies needed for mobile operators?", 21st European Regional ITS Conference, September 13-15, 2010, Copenhagen, Denmark.

[24] T. Giles, J. Markendahl, J. Zander, P. Zetterberg, P. Karlsson, G. Malmgren, and J. Nilsson, "Cost drivers and deployment scenarios for future broadband wireless networks-key research problems and directions for research", IEEE Vehicular Technology Conference (VTCSpring 2004), May 17-19, 2004, Milan, Italy.

[25] http://tet.sec-salus.eu/

[26] T. Chrysikos and S. Kotsopoulos, "Site-specific validation of the path loss models and large-scale fading characterization of large-scale fading for a complex urban propagation topology at $2.4 \mathrm{GHz}$ ", 2013 IAENG International Conference on Communication Systems and Applications (IMECS 2013), March 13-15, 2013, Hong Kong.

[27] T. Chrysikos and S. Kotsopoulos, "Characterization of large-scale fading for the $2.4 \mathrm{GHz}$ channel in obstacle-dense indoor propagation topologies", IEEE Vehicular Technology Conference (VTC-Fall 2012), September 3-6, 2012, Quebec City, Canada.

[28] P. Vieira, M.P. Queluz, and A. Rodrigues, "LTE Multi Antenna Bit Rate Expectation for Urban Macro-cell Networks", 7th Ibero-American Congress on Sensors (IBERSENSOR 2010), November 9-11, 2010, Lisbon, Portugal.

[29] P. Vieira, M.P. Queluz, and A. Rodrigues, "LTE Spectral Efficiency using Spatial Multiplexing MIMO for Macro-cells", 2nd IEEE International Conference on Signal Processing and Communication Systems (ICSPCS 2008), December 15-17, 2008, Gold Coast, Australia. 